\title{
A Cloud Platform for Smart Firefighting Facilities Maintenance Based Internet of Things
}

\author{
Wenchao Guan ${ }^{1}$, Jianjun $\mathrm{Yi}^{1, *}$, Liang $\mathrm{He}^{2,3}$,Yajun Zhang ${ }^{1}$ and Pengfei Liu ${ }^{2,3}$ \\ ${ }^{1}$ Department of Mechanical Engineering, East China University of Science and Technology, Shanghai 200237, China; \\ ${ }^{2}$ Shanghai Aerospace Control Technology Institute, Shanghai 201109, P. R. China; \\ ${ }^{3}$ Shanghai Key Laboratory of Aerospace Intelligent Control Technology, Shanghai 201109, P. R. China \\ *Corresponding author
}

\begin{abstract}
The traditional form of maintenance and management of fire facilities has shortcomings such as low efficiency, inadequate supervision, inaccurate updating and inconvenient query of information, causing huge security risks. Therefore, this paper proposes and designs an intelligent maintenance cloud platform for fire facilities based on Internet of things (IoT) and cloud computing, to realize the centralization and transparency of maintenance and management of fire facilities. The platform mainly consists of three subsystems, task management system, facilities file management system and information query system, which archives those functions like the generation, dispatching and management of maintenance task, fire-fighting facilities archives management and online statistic query. Moreover, it provides users with SaaS services in the form of web and app, which reduces the user's investment in the construction of data center and the development of software. After deployment and operation in a construction unit, the result shows that this platform greatly facilitates the maintenance management of firefighting facilities, improves maintenance efficiency and reduces maintenance cost.
\end{abstract}

Keywords-IoT; fire-fighting maintenance; SaaS; cloud computing

\section{INTRODUCTION}

The traditional firefighting facilities maintenance is mainly in the form of manual inspection, manual records, paper-based archives, resulting in inefficient supervision, facilities monitoring oversight, the information update delay, inconvenience query of the fire information and many other issues. As a result, the inspection for firefighting facilities is frequently missing and the available firefighting equipment cannot be found in the event of a disaster, which leads to a huge security risk [1]. In addition, single, scattered maintenance system is not conducive to the supervision of fire management. Therefore, it is imperative to study a centralized and platform-based system to achieve fire maintenance management.

Internet of things technology expands the Internet application to the real object and realizes the identification, location, monitoring and management of everything through the collection, transmission, exchange, processing and other processes of physical information. Therefore, by using Internet of things technology, it's possible to identify every fire facility, collect the level, water pressure, voltage and switch volume of fire facilities, and realize the real-time perception of fire facilities [2]. However, in the face of the storage processing of massive data and the problem of rapid deployment and iteration of the system, the traditional system architecture is not enough to deal with. Thus, it's necessary to introduce cloud-computing technology, processing massive data collected by Internet of things, and carrying Internet of things system, to provide users with platform services.

At present, the firefighting maintenance systems at home and abroad are being developed and in operation, but most of these are independent systems. They cannot provide platform services and easily lead to forming information islands. Therefore, based on Internet of Things and cloud computing technology, this paper designs and develops a cloud platform for smart firefighting facilities maintenance, to provide platform services for the maintenance and avoid shortcomings of current systems. The main contents are as follows: the first part mainly introduces design of this cloud platform architecture, including basic IoT layer and platform layer, the second part focuses on the design and implementation of main functions of intelligent maintenance system operating in this cloud platform. Finally, this paper introduces the verification of this platform and makes a conclusion.

\section{ARCHITECTURE OF THE PlATFORM}

To achieve intelligent maintenance of firefighting facilities, it needs to identify every facility and record the data including basic information and maintenance records so that we can obtain real-time operating status of fire-fighting facilities, to find out the problems in the operation of fire-fighting facilities in time and eliminate the hidden danger in the bud. Therefore, the cloud platform for smart fire facilities maintenance designed by this paper consists of IoT infrastructure layer and cloud platform layer. Figure I shows the architecture diagram. 


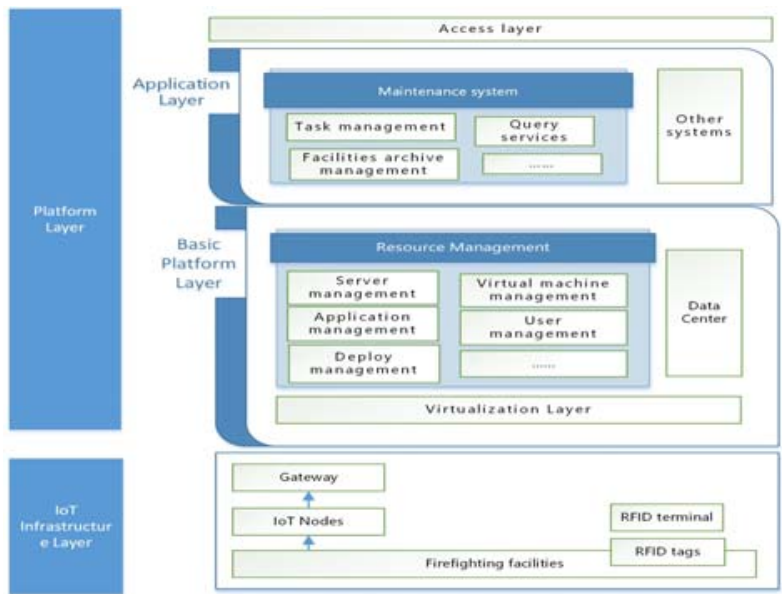

FIGURE I. THE PLATFORM ARCHITECTURE

In the IoT infrastructure layer, real-time on-line monitoring of firefighting facilities can be accomplished by deploying self-developed IoT monitoring nodes to collect firefighting facilities data such as water pressure and power supply in real time, and then forwarding the data to the cloud platform, so that abnormal situation can be found and processed in time. RFID tags are used to identify firefighting facilities, and the RFID terminal can upload the real-time data like the data of facilities maintenance so as to achieve accurate and timely collection and tracking of facilities data.

In the platform layer, the support layer provides the basic environment for the application layer and is divided into three layers, virtualization layer, resource management layer and data center. The virtualization layer virtualizes physical resources including server clusters, storage devices and network devices and so on to form a virtualization pool $[3,4]$. The resource management layer is responsible for all virtual resource management, including resource allocation, virtual machine resource management, application deployment, user management and so on. And the data center is responsible for storing and processing data related to the platform and cloud applications [5]. The application layer carries the system of smart firefighting facilities maintenance designed by this paper and provides firefighting maintenance services in SaaS for users.

\section{DESIGN AND IMPLEMENTATION OF THE PlATFORM}

The cloud platform for smart firefighting facilities maintenance designed by this paper mainly provides users with SaaS form of maintenance service. Therefore, public cloud is used for providing cloud platform operation environment for application platform layer. And in the application platform layer, services are mainly provided by the smart firefinghting maintenance system, which constructed by J2EE technology [6]. The architecture diagram of this system is shown in Figure II.

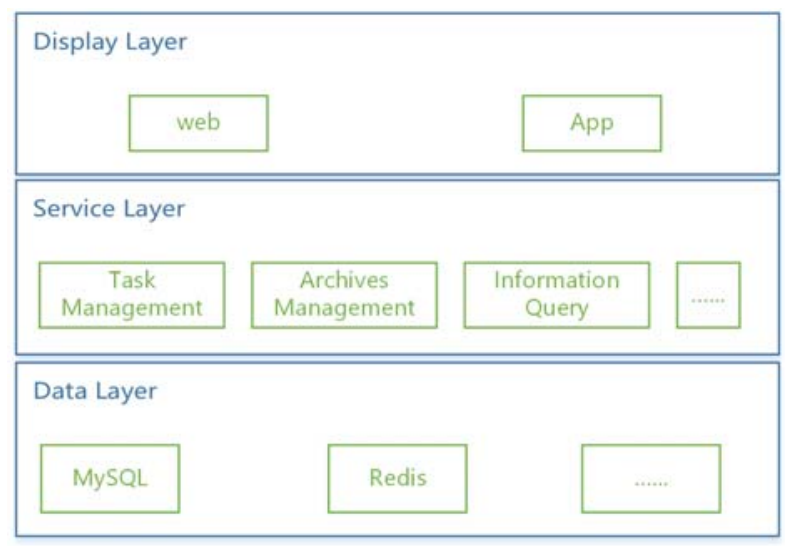

FIGURE II. SMART MAINTENANCE SYSTEM ARCHITECTURE

\section{A. Task Management Subsystem}

The task management subsystem is mainly responsible for the configuration, generation, scheduling and management of tasks such as the maintenance task and the inspection task. This system consists of four parts: application service for maintenance, task configuration service, task scheduling service and task management service. Figure III shows data stream between above four parts.

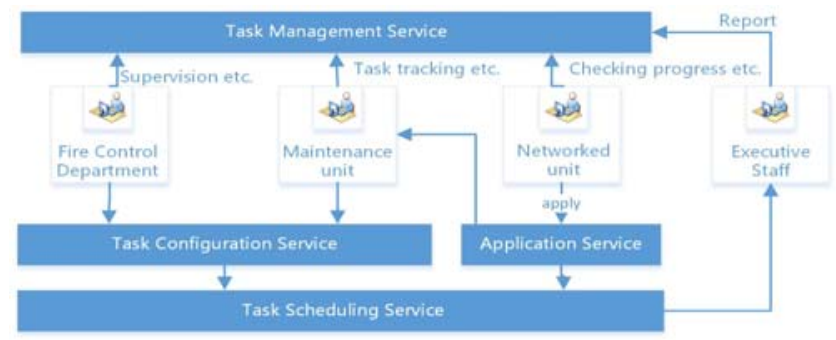

FIGURE III. THE RELATIONSHIP DIAGRAM OF FOUR PARTS OF TASK MANAGEMENT SUBSYSTEM

The task configuration service provides configuration service for different types of task, including periodic task, task from application form, random task and so on. The firefighting maintenance unit can configure periodic maintenance tasks, inspection tasks through the service, including 2-hours inspection 8-hours inspection, daily inspection, weekly inspection and custom inspection and so on. And the fire management unit can create random inspection task to complete the fire safety inspection work for the jurisdiction units throw the service. After the configuration completed, the system automatically generates the task book and sends it to the scheduling system for unified task scheduling.

The task scheduling service part mainly achieves the function of dispatching tasks to the corresponding personnel, including maintenance application, periodic tasks, tasks that need to be manually scheduled, and tasks that are temporarily scheduled. When the user's application for maintenance service is approved, the dispatching service will select the appropriate maintenance personnel to receive the task according to the priority of the task and the location of the service client and the location of the service personnel on the map, and send the task to 
the selected maintenance personnel. Figure IV shows the workflow of this service.

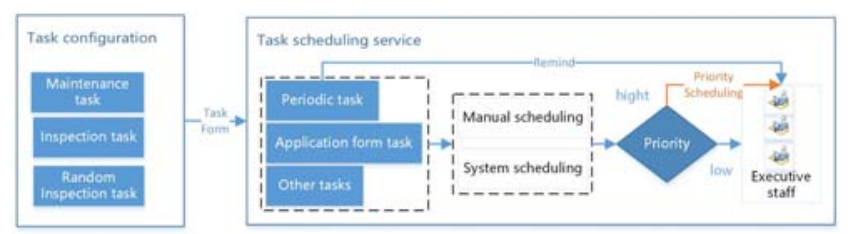

FIGURE IV. THE WORKFLOW OF TASK SCHEDULING SERVICE

The task management service provides services with users like tracking position and status of workers during the task, checking task progress, reviewing task reports and evaluating maintenance service and so on. During task execution, the system tracks the real-time geographic location of the workers corresponding to the task and records the real-time trajectories and the task logs. After the task completed, the system automatically generates the task report according to the task records uploaded by the operator for the relevant personnel to review. The networked unit users can make evaluation for the maintenance service, which will be included in the task report and checked by the audit staff. Figure V shows the service workflow.

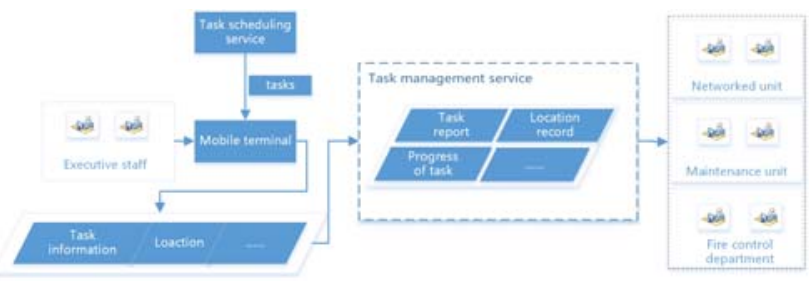

FIGURE V. THE WORKFLOW OF TASK MANAGEMENT SERVICE

\section{B. Management Subsystem of Facilities Archives}

The management subsystem of facilities archives is responsible for the management of electronic archives of firefighting facilities. First of all, by sticking or packaging RFID tags on fire-fighting facilities they can get the unique identification mark. These tags can only be read or written by the RFID terminals that have been registered in the platform, which solves the problem of difficult identification of fire-fighting facilities. Then, users can use those terminals to record and upload the usage of firefighting equipment or the data of maintenance or inspection of firefighting facilities by reading or writing the tags on them $[2,7,8]$. These data will be uploaded into this subsystem. When there are abnormal information such as abnormal report, expiration of firefighting equipment, the system will automatically notify the manager to process, ensuring that all the equipment is in the available state. In this way, the system can record and track all the data in firefighting facilities service cycle.

\section{Information Query Subsystem}

This paper builds query engine for the information query subsystem based on Elastic Search, which provides query services to user information, statistical information, and historical record and so on:
- User information query: This service provides user account detail and basic information of all networking units. These information will be different for different roles.

- Statistical query: The statistics query service provides diversified data statistics service and displays the query results graphically. And different users can get different query result according to their authorities. For example, networking units can just make statistical inquiries on the operation of their own firefighting facilities and keep abreast of the operation of fire-fighting facilities. The fire control departments can make statistical query on all the data in the area under their jurisdiction.

- History query: The history query service provides querying of the history of maintenance tasks, the inspection tasks and other historical records, including the application form of maintenance service, service job records of service personnel, job reports, and the history of job scheduling records and so on.

\section{VERIFICATION OF THE PlATFORM}

This paper takes the firefighting facilities maintenance in a certain unit's building as a pilot to verify the function and stability of the system. Take fire extinguisher maintenance in this unit as an example. The management of the unit fire extinguisher is chaotic before access to the system. The problems like lack of information records, lack of maintenance, fire extinguishers missing, expired issues not found in time etc. existed for a long time. After access to the system, the fire extinguishers in all places are identified through RFID tags and their electronic records are stored into the data center of the platform. By scanning these tags through mobile RFID terminals, the user can query detail information of a fire extinguisher, record and upload the abnormal information or data of fire extinguishers inspection or maintenance, which ensures in-time update of relevant information of the fire extinguisher. By summarizing all these information, the system feedback problems such as fire extinguishers missing or expiring to the responsible person in time to guarantee that they can identify and solve these problems in time. After online configuration of periodic inspection of fire extinguisher and maintenance tasks, the system automatically sends these tasks to designated personnel and reminds them when the time of next execution of the task is up. It ensures the effective implementation of fire extinguisher maintenance system, avoiding the problem of missing even disorderly, giving to the unit a great convenience of fire extinguisher maintenance management work. Figure VI shows a pc webpage screenshot of the system. 


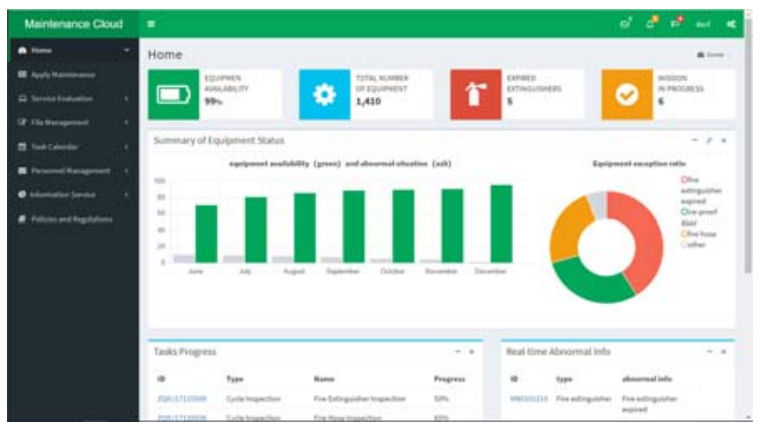

FIGURE VI. HOMEPAGE SCREENSHOT OF SYSTEM UNDER THE AUTHORITY OF NETWORKED UNIT

Through the pilot verification in this unit, it shows that this cloud platform designed by this paper can provide users with platform-based maintenance services, greatly improving the maintenance efficiency of fire facilities and reducing costs, achieving the anticipated goals.

\section{CONCLUSION}

This paper, by combining Internet of things and cloud computing technology, designs and develops a cloud platform for smart firefighting facilities maintenance, which provides platform services for the maintenance and management. By using this platform, users of networked units can conveniently apply, track and evaluate maintenance services, and the maintenance units can promptly conduct the acceptance, arrangement and management of maintenance services. In addition, fire control departments can conveniently conduct relevant inspections, issue announcements. It greatly improves the efficiency of fire maintenance and management and reduces costs. Morever, the design of this platform fully takes into account the versatility and scalability so that the platform can be applied to not only the fire facilities maintenance, but also other facilities maintenance field.

\section{ACKNOWLEDGMENTS}

This paper was supported by the Military and civilian integration project(Shanghai economic and information commission) under Grant No.201720, the Natural Science Fund of China (NSFC) under Grant Nos. 51575186, 51275173, and 50975088, the Fundamental Research Funds for the Central Universities under Grant No. WH0913009, Shanghai Pujiang Program under Grant No. PJ201000353, and Shanghai Software and IC industry Development Special Fund under Grant No. 120493.

\section{REFERENCES}

[1] Tian-yi Shen, Xing-yuan Wang, Nan Xu, Qian Cao. The design of firefight maintenance supervision system by information technology, J. Electronic Technology \& Software Engineering. 12 (2017) 228.

[2] Lei Xu. Research on Smart Grid Equipment Management System based on RFID Internet of things Technology, D. North China Electric Power University. (2016).

[3] Fu-yong Kang. Environmental Private Cloud Platform Design and its SaaS Model Zongliang Jianpai Business System Implementation, D. University of Electronic Science and Technology of China. (2013).
[4] Jin-Mook Kim, Jeong-Kyung Moon, Bong-Hwa Hong. Research of Virtualization Services Valuation for Cloud Computing Environment, J. Embedded and Multimedia Computing Technology and Service. 181 (2012) 631-620.

[5] Harrabi M A, Sofiene C, Wafa M. Deploying an internet of things cloud platform on openstack, J. Information Technology and Computer Applications Congress. (2015).

[6] Cheng-gang Yang. Fire Management System Design and Implementation Based on Internet of Things, D. Jilin University. (2015).

[7] Zhong-quanYang. Design and Realization of Firefighting Facilities Monitoring Platform Based on RFID Technology, J. Technology and Economic Guide. 20 (2016) 18-19.

[8] Na Li, Ai-nan Wang. Intelligent Fire Storage System Based on Internet of Things, J. Internet of things technologies. 11 (2017) 42-44. 